\title{
Von Gauß bis LIGO
}

\author{
Thomas Vogt
}

Am 6. November 2020 fand auf Anregung und in Zusammenarbeit mit der Wissenschaftsabteilung der Französischen Botschaft im Rahmen der Berlin Science Week, der Falling Walls Conference Berlin, der Année des Mathématiques des CNRS und von 130 Jahre DMV in Berlin ein Online-Kolloquium zu „Mathematik und Kosmologie“ statt. Zwei Vorträge suchten Antworten auf die Frage „Why did Einstein's equation change completely our understanding of gravity?"

Thomas Simon, promovierter Mathematiker und Wissenschaftsattaché in der Französischen Botschaft, eröffnete die Veranstaltung und stellte die Redner und die Moderatorin vor. Als Erster sprach Jean-Pierre Bourguignon, Professor für Mathematik am CNRS und Präsident des Europäischen Forschungsrats ERC. Er beleuchtete das Thema aus mathematischer und historischer Perspektive, indem er zunächst die innovativen Gedanken von Carl Friedrich Gauß und Bernhard Riemann zur Geometrie des Raums und dem Begriff der Krümmung Mitte des 19. Jahrhunderts skizzierte und dann Albert Einsteins Arbeiten zur Elektrodynamik bewegter Körper (1905) vorstellte. Anschließend erläuterte er Albert Einsteins und Marcel Grossmanns Entwurf einer verallgemeinerten Relativitätstheorie und einer Theorie der Gravitation aus dem Jahr 1913, wobei Einstein für den physikalischen Teil verantwortlich zeichnete und Grossmann für den mathematischen; dabei wurde deutlich, wie eng die physikalische Interpretation des Gravitationsfeldes mit seiner mathematischen Modellierung als Metrik verknüpft ist. Folgerichtig war es der Mathematiker David Hilbert, der darauf aufbauend die Einstein-Gleichung aus einem Variationsprinzip herleitete (1915).

Danach sprach Julien Lesgourgues, Professor für Theoretische Astroteilchenphysik und Kosmologie an der Rheinisch-Westfälischen Universität Aachen (RWTH) über die Errungenschaften der modernen Forschung im Bereich Astrophysik, die schlussendlich zum experimentellen Nach- weis von Einsteins theoretischer Vorhersage von Gravitationswellen führten. Lesgourgues ließ zunächst einige Meilensteine der astrophysikalischen Forschung Revue passieren. Dann kam er auf die Ausbreitung von Gravitationswellen, bedingt durch die schnelle Rotation sehr massereicher Körper, zu sprechen. Gravitationswellen stellen einen eigenen fundamentalen Wellentyp dar, der zwar Analogien zu elektromagnetischen Wellen besitzt, aber eine Herausforderung an die physikalische Anschauung sind: Nicht Objekte schwingen im Raum, sondern das Gravitationsfeld selber und mit ihm der Abstand zwischen zwei festen Punkten (der dann eben nicht mehr konstant ist) und die Raumkrümmung. Der experimentelle Nachweis gelingt über hochsensible Laser-Interferometer, insbesondere LIGO (USA) und VIRGO (Europa). Diese fangen die schwachen Schwingungen von schnell rotierenden Neutronensternen oder Schwarzen Löchern auf. Diese experimentelle Leistung wurde 2017 mit dem Nobelpreis für Physik geehrt.

Nach den Vorträgen gab Ilka Agricola, Professorin für Differentialgeometrie und Analysis an der PhilippsUniversität Marburg, Mitglied des DMV-Präsidiums und designierte DMV-Präsidentin für 2021/22, die zwischenzeitlich von den Teilnehmer*innen im Livechat gestellten Fragen zusammengefasst an die Redner weiter, die sie dann im Wechsel beantworteten. Während der Konferenz waren über 1 oo Personen online. Sie bedankten sich am Ende der Veranstaltung mit enthusiastischem Applaus.

Thomas Vogt, Medienbüro Mathematik, Freie Universität Berlin, Institut für Mathematik, Königin-Luise-Straße 24-26, 14195 Berlin Tel. (o3o)83875657·medienbuero@mathematik.de

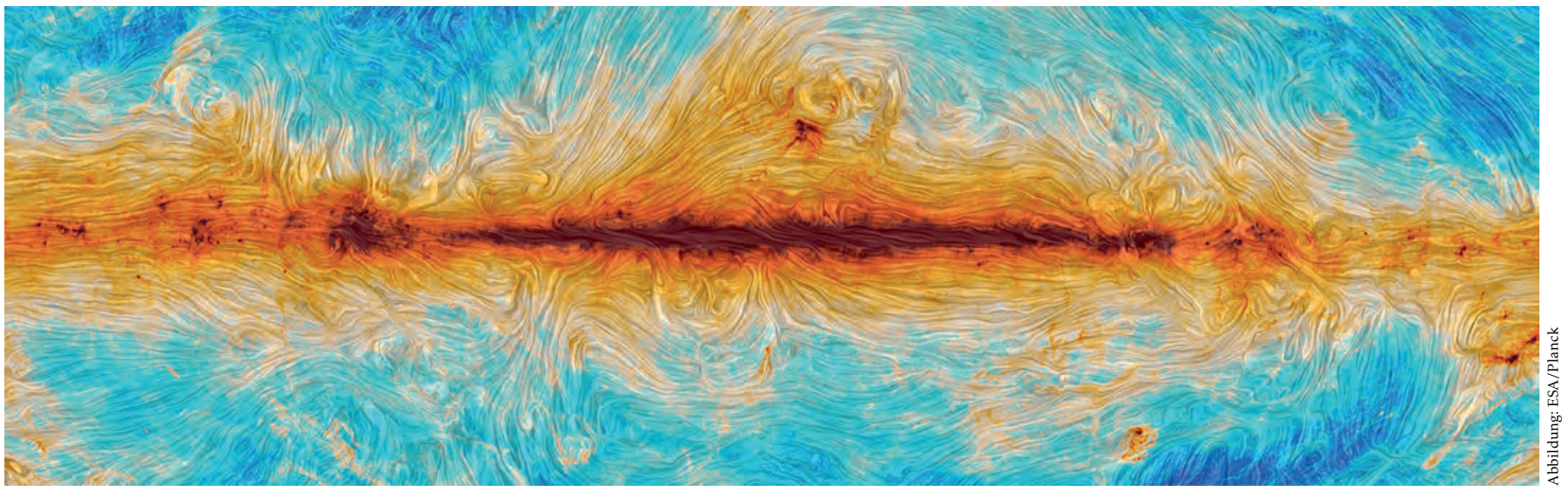

Karte des Magnetfeldes unserer Galaxie 